\title{
Mono-parametric charge pumping through a quantum dot coupled with energy-gapped leads
}

\author{
Tomasz Kwapiński ${ }^{\mathrm{a}}$ and Ryszard Taranko \\ Institute of Physics, M. Curie-Skłodowska University, 20-031 Lublin, Poland
}

Received 6 February 2015 / Received in final form 24 April 2015

Published online 1st June 2015

(C) The Author(s) 2015. This article is published with open access at Springerlink.com

\begin{abstract}
We present a proposal for a single-parametric electron pump composed of a quantum dot between two unbiased leads with energy-gapped electron density of states (DOS). The model tight-binding Hamiltonian and the evolution operator technique are used in the calculations. The quantum dot is driven by the external harmonic field which leads to the pumping current flowing from the left or right electrode depending on the system parameters. We show that the net pumping current appears in the system if (i) there are at least two sideband states: one of them lying below and the second lying above the Fermi energy; (ii) the left and right lead DOS in the vicinity of these sideband states are different. Moreover, the energy-gapped structure of DOS is visible on the average quantum dot charge and the pumped current curves as well as on the transconductance characteristics. Thus mono-parametric pumping provides useful information about the system parameters, in particular about the lead DOS structure.
\end{abstract}

\section{Introduction}

The phenomenon of electron pumping in low-dimensional systems has attracted considerable attention recently due to its potential applications in spintronics, quantum computing or metrology (quantum current standard). Many interesting effects were found in quantum dot (QD) systems driven by external time-dependent forces like the turnstile effect, photon-assisted tunnelling (PAT), spin and charge quantum pumps, e.g. [1-7]. For periodically modulated quantum system a net number of charge can be transferred between unbiased electrodes. In the adiabatic limit one needs at least two time-dependent forces to generate the net pumping current [8]. In this case periodic changes of the device-control parameters (tunnelling barriers, gate voltages) break the spatial symmetry of the system which leads to the charge pumping effect. Such pumps have been realized experimentally e.g. for $\mathrm{QD}$ or double-QD (double-atom) systems [2,5,9-11]. Also onedimensional quantum structures in the presence of periodic potentials applied to two sites were proposed as charge pumps [12-16]. For more complex systems adiabatic quantum pumps have been studied as well, e.g. graphene pumps with two vibrating or oscillating potential barriers [17-19]. Moreover, spin and charge pumping can occur in low-dimensional systems due to the Zeeman splitting, spin-orbit interactions or in the presence of external train-like impulse (which influences the coupling parameters in the system) [5,20-22]. Such a train impulse

\footnotetext{
a e-mail: tomasz.kwapinski@umcs.pl
}

can be generated by acoustic waves which spread through e.g. carbon nanotubes [23].

The pump current can be also generated by external time-dependent fields in the case of a lack of time-reversal symmetry in the ac signal. It holds e.g. for bi-harmonic fields (the second harmonic is added to the driving periodic potential) or for time-dependent dipole forces [24-26]. For the asymmetric double-well heterostructure in the presence of harmonic (dipole-like) modulations applied to both wells a heat pump was predicted which could operate at zero electron current [27]. Recently, also mono-parametric charge pumping devices have been studied both theoretically and experimentally [28-37]. Such pumps need only one external harmonic field applied to the unbiased system and thus they are of great interest. Note that it is technologically easier to fabricate a pump with only one external time-dependent source but more important it involves a reduction of the system size due to smaller number of contacts. In consequence one expects also a reduction of dissipation processes in comparison with many-parameter pumps. Moreover, single-parameter pumping does not rely on a phase shift (between external time-dependent perturbations) which appears as additional system parameter for dipole-like forces or, in general, in two-parameter pumping. It is worth noting that the driving by changing of only one parameter excludes the emergence of adiabatic pumping thus only non-adiabatic transport can appear in mono-parametric pumps. However, in the presence of the left-right spatial symmetry, periodical changes of only one system parameter cannot generate the net current (dipole-like forces applied e.g. to the double-QD system are in fact two separate 
signals which modulate each QD in different ways). Considering that spatial symmetry is broken, e.g. in the presence of magnetic fields which changes the QD-lead couplings [28] or by tuning the static gate voltages [24,38], the pumping current can appear in the system. Note that single-parameter efficient quantum pumps can be realized for different systems e.g. QDs, graphene, carbon nanotubes and nanoribbons [29-31,33-35,37]. However, periodically driving of only one system parameter (e.g. harmonic modulation of the QD-lead tunnelling barrier in experiments [29-31]) often disturbs other parameters (the positions of the QD energy levels or, in general, the shape of the QD well). Thus effectively, these systems are two-parameter electron pumps.

In this paper we consider mono-parametric pumping through a single QD between two unbiased leads (metallic and semiconductor-like). Our new proposal is to generate the pumping current in the presence of an energy gap in the lead density of states (DOS). We assume that the left and right electrodes are characterized by different DOS: one of them is described by the structure-less flat DOS and the second one contains an energy gap (or a pseudo-gap) in the middle of its DOS structure. Different DOS structures of the left and right leads break the spatial symmetry of the system and thus a net current can arise for non-adiabatic mono-parametric driving. From the experimental point of view such studies are important because in real quantum systems electron reservoirs are often characterized by nontrivial band structures i.e. with peaks, gaps or surface states (see e.g. STM experiments on semiconductor surfaces). Therefore the lead DOS structure plays a crucial role in the electron transport in low dimensional systems. Note that single electron tunnelling in energy-gapped materials were measured experimentally e.g. for superconducting leads [32] or semiconductor nanowires [31]. In this work we realize a monoparametric charge pump and investigate the role of the lead DOS structure on the electron pumping effect. Additionally, in our studies we show that it is possible to determine some parameters of the system (e.g. energy values of the gap edges) directly from the charge or pumped current characteristics.

In our calculations we use a time-dependent tightbinding Hamiltonian and the evolution operator method which was successfully applied for arbitrary time dependence of the external perturbations, e.g. [39-42]. The problem we study here is relatively complex because we consider the time-dependent transport through the system with energy-gapped band structure of the leads. In the case when the lead's DOS varies slowly with energy (in the vicinity of the levels of the central scattering region) a wide band approximation (WBL) can be used with the energyindependent spectral density function [43]. This procedure drastically simplifies mathematical derivations which often leads to analytical formulas for the current or $\mathrm{QD}$ charge in the stationary as well as time-dependent processes [24,40,42-44]. However, electron reservoirs are often characterized by complex band structures which need nontrivial calculations beyond the WBL approximation especially for time-dependent forces acting on the system, e.g. $[42,45,46]$.

The paper is structured as follows. In Section 2 we briefly present the Hamiltonian and the calculation method. In Section 3 we show and discuss the numerical results of the pumping current and the QD occupations for the system with an energy gap. The last section is devoted to conclusions.

\section{Model and theoretical description}

Our model consists of a QD which is coupled through the tunnelling barriers with the left and right leads. The right lead possesses an energy gap (pseudo-gap) in its DOS structure, similarly to semiconductor-like materials. By a pseudo-gap (named here also the gap) we mean the energy region in the lead DOS with very small values in comparison with the non-gapped DOS structure of the lead. It allows us to avoid bonding states in the system which can appear beyond the lead energy bands [47] and concentrate on the electron pumping effect. We consider our system in the presence of the external microwave field applied to the QD inducing the time-dependence of the dot energy level. For simplicity the dot is characterized by a single level $\varepsilon_{d}$ and the intradot Coulomb interaction is neglected. The Hamiltonian of the system can be written in the second quantization notation:

$$
H=H_{\text {leads }}+H_{Q D}(t)+V(t),
$$

where $H_{\text {leads }}=\sum_{k, \alpha=L, R} \varepsilon_{k \alpha} c_{k \alpha}^{+} c_{k \alpha}, H_{Q D}(t)=\varepsilon_{d}(t) c_{d}^{+} c_{d}$ and $V(t)=\sum_{k, \alpha=L, R} V_{k \alpha}(t) c_{k \alpha}^{+} c_{d}+$ h.c. Here $c_{k \alpha}\left(c_{k \alpha}^{+}\right)$, $c_{d},\left(c_{d}^{+}\right)$are the electron annihilation (creation) operators in the $\alpha$ th lead and in the dot, respectively. The electron tunnelling between the dot and leads is described by $V_{k \alpha}$ and $\varepsilon_{k \alpha}$ denotes the electron energy spectrum of the $\alpha$ th lead. We assume that the dot energy level is driven harmonically in time, $\varepsilon_{d}(t)=\varepsilon_{0}+\Delta \cos (\omega t)$, with the driving amplitude $\Delta$ and the microwave frequency $\omega$ which is smaller than the plasma frequency of the leads. Thus the influence of the ac field on the leads is screened perfectly [43].

The time evolution of the QD charge and the current flowing between leads and the dot is described in terms of the time-evolution operator $U\left(t, t_{0}\right)$ given (in the interaction representation) by the equation $(\hbar=1)[39,40,42]$ :

$$
i \frac{d U\left(t, t_{0}\right)}{d t}=U_{0}\left(t, t_{0}\right) V(t) U_{0}^{+}\left(t, t_{0}\right) U\left(t, t_{0}\right),
$$

where $U_{0}\left(t, t_{0}\right)=T \exp \left(i \int_{t_{0}}^{t} d t_{1}\left(H_{\text {leads }}+H_{Q D}\left(t_{1}\right)\right)\right)$ and $T$ denotes the time ordering. We assume also that the interaction between leads and QD is switched on at $t_{0}=0$ such that $V_{k \alpha}(t)=V_{\alpha}(t)=0$ for $t<0$ and $V_{k \alpha}(t)=V_{\alpha}$ for $t \geq 0$. The charge localized on the QD, $n_{d}(t)$, can be expressed by the matrix elements of the evolution operator:

$$
n_{d}(t)=n_{d}\left(t_{0}\right)\left|U_{d, d}\left(t, t_{0}\right)\right|^{2}+\sum_{k, \alpha=L, R} n_{k \alpha}\left(t_{0}\right)\left|U_{d, k \alpha}\left(t, t_{0}\right)\right|^{2}
$$


where the basis set of functions containing the singleparticle states $|k \alpha\rangle,|d\rangle$ was used and $U_{i j}=\langle i|U| j\rangle$.

The tunnelling current flowing e.g. from the left lead, $j_{L}(t)$, can be obtained from the formula $j_{L}(t)=$ $-e \frac{d n_{L}(t)}{d t}$ [43], where $n_{L}(t)$ denotes the total number of electrons in the left lead:

$$
\begin{aligned}
n_{L}(t)= & n_{d}\left(t_{0}\right) \sum_{k}\left|U_{k L, d}\left(t, t_{0}\right)\right|^{2} \\
& +\sum_{k, k_{1}, \alpha=L, R} n_{k_{1} \alpha}\left(t_{0}\right)\left|U_{k L, k_{1} \alpha}\left(t, t_{0}\right)\right|^{2} .
\end{aligned}
$$

After some algebra, using equation (2), the formula for the current reads:

$$
\begin{aligned}
j_{L}(t)= & -2 e \operatorname{Re}\left\{V_{L}^{2} n_{d}\left(t_{0}\right) U_{d, d}\left(t, t_{0}\right)\right. \\
& \times \int_{t_{0}}^{t} d t_{1} \tilde{D}_{L}\left(t, t_{1}\right) U_{d, d}^{*}\left(t_{1}, t_{0}\right) \\
& +\sum_{k L} n_{k L}\left(t_{0}\right) \tilde{V}_{k L}(t) U_{d, k L}\left(t, t_{0}\right) \\
& +\sum_{k, \alpha=L, R} V_{L}^{2} n_{k \alpha}\left(t_{0}\right) U_{d, k \alpha}\left(t, t_{0}\right) \\
& \left.\times \int_{t_{0}}^{t} d t_{1} \tilde{D}_{L}\left(t, t_{1}\right) n_{k \alpha}\left(t_{0}\right) U_{d, k \alpha}^{*}\left(t_{1}, t_{0}\right)\right\} .
\end{aligned}
$$

Here

$$
\begin{aligned}
\tilde{D}_{\alpha}\left(t, t_{1}\right)= & D_{\alpha}\left(t-t_{1}\right) \exp \left(-i \varepsilon_{0}\left(t-t_{1}\right)\right) \\
& \times \exp \left(-i \frac{\Delta}{\omega}\left(\sin (\omega t)-\sin \left(\omega t_{1}\right)\right)\right),
\end{aligned}
$$

where $D_{\alpha}(t)=\int d \varepsilon D_{\alpha}(\varepsilon) \exp (i \varepsilon t)$ and $D_{\alpha}(\varepsilon)$ is the electron density of states in the $\alpha$ th lead. The matrix elements $U_{d, d}\left(t, t_{0}\right)$ and $U_{d, k \alpha}\left(t, t_{0}\right)$ satisfy the following integrodifferential equations (obtained from Eq. (2)):

$$
\begin{aligned}
\frac{d U_{d, d}\left(t, t_{0}\right)}{d t} & =-\int_{t_{0}}^{t} d t_{1} K\left(t, t_{1}\right) U_{d, d}\left(t_{1}, t_{0}\right) \\
\frac{d U_{d, k \alpha}\left(t, t_{0}\right)}{d t} & =-i \tilde{V}_{k \alpha}(t)-\int_{t_{0}}^{t} d t_{1} K\left(t, t_{1}\right) U_{d, k \alpha}\left(t_{1}, t_{0}\right),
\end{aligned}
$$

where $\tilde{V}_{k \alpha}(t)=V_{\alpha} \exp \left(-i\left(\varepsilon_{0}-\varepsilon_{k \alpha}\right) t-i \frac{\Delta}{\omega} \sin (\omega t)\right)$ and $K\left(t, t_{1}\right)=\sum_{k, \alpha=L, R} \tilde{V}_{k \alpha}^{*}(t) \tilde{V}_{k \alpha}\left(t_{1}\right)$ stands for the integral kernel. The solutions of equations (7) and (8) are inserted into the formulas for the occupation $n_{d}(t)$ and the current $j_{L}(t)$. Because the driving field is periodic in time we averaged $n_{d}(t)$ and $j_{L}(t)$ over the period (the averages are noted $\langle n(t)\rangle$ and $\left\langle j_{L}(t)\right\rangle$, respectively) and for the averaged currents the following relation is always satisfied $\left\langle j_{L}(t)\right\rangle=-\left\langle j_{R}(t)\right\rangle$. Note that in the high-frequency limit or in the case of vanishing time-dependent field, $\Delta \rightarrow 0$, the exact analytical solutions for $U_{d, d}\left(t, t_{0}\right)$ and $U_{d, k \alpha}\left(t, t_{0}\right)$ can be obtained using e.g. the Laplace transformation technique. In that case, equations (7) and (8) are the integro-differential equations with convoluted kernels $\int_{t_{0}}^{t} d t_{1} K\left(t-t_{1}\right) U_{d, d(d, k \alpha)}\left(t_{1}, t_{0}\right)$ for which the Laplace transforms exist. For example, taking the electron DOS of both leads in the form of the Lorentzian functions $D_{L / R}(\varepsilon)=\frac{2}{\pi} \frac{\beta}{\varepsilon^{2}+4 \beta^{2}}$ and assuming $V_{L}=V_{R}=V, t_{0}=0$, the function $U_{d, d}\left(t, t_{0}\right) \equiv U_{d, d}(t)$ satisfies the following equation:

$$
\frac{d U_{d, d}(t)}{d t}=-2 V^{2} \int_{0}^{t} d t_{1} \exp \left(-\left(t-t_{1}\right)\left(2 \beta-i \varepsilon_{0}\right)\right) U_{d, d}\left(t_{1}\right)
$$

for which the Laplace transform reads:

$$
U_{d, d}(s)=\frac{s-s_{3}}{\left(s-s_{1}\right)\left(s-s_{2}\right)}
$$

where $s_{3}=i \varepsilon_{0}-2 \beta$ and $s_{1}, s_{2}$ are the solutions of $s(s+$ $\left.2 \beta-i \varepsilon_{0}\right)+2 V^{2}=0$ and $4 \beta$ is the electrode bandwidth. Finally we obtain

$$
U_{d, d}(t)=\frac{1}{s_{1}-s_{2}}\left(\left(s_{1}-s_{3}\right) e^{s_{1} t}-\left(s_{2}-s_{3}\right) e^{s_{2} t}\right),
$$

and e.g. for $\varepsilon_{0}=0$ it takes the form (cf. also [48])

$$
\begin{aligned}
U_{d, d}(t)= & \frac{e^{-\beta t}}{\sqrt{2 V^{2}-\beta^{2}}}\left(\beta \sin \sqrt{2 V^{2}-\beta^{2}} t\right. \\
& \left.+\sqrt{2 V^{2}-\beta^{2}} \cos \sqrt{2 V^{2}-\beta^{2}} t\right) .
\end{aligned}
$$

Similar calculations can be also done for other matrix elements. In the case of non-vanishing microwave field, due to the exponential term $\exp \left(i \frac{\Delta}{\omega}\left(\sin (\omega t)-\sin \left(\omega t_{1}\right)\right)\right)$ included in equations (7) and (8), the Laplace transform of e.g. $\tilde{U}_{d, d}(t) \equiv e^{-i \frac{\Delta}{\omega} \sin (\omega t)} U_{d, d}(t)$ forms the algebraic equation with displaced arguments. It is a consequence of the time-dependent coefficient appearing in the first term on the right-hand side in the integro-differential equation

$$
\begin{aligned}
\frac{\tilde{d}_{d, d}(t)}{d t}= & -i \Delta \cos (\omega t) \tilde{U}_{d, d}(t) \\
& -2 V^{2} \int_{0}^{t} d t_{1} \exp \left(-\left(t-t_{1}\right)\left(2 \beta-i \varepsilon_{0}\right)\right) \tilde{U}_{d, d}\left(t_{1}\right) .
\end{aligned}
$$

Thus it is impossible to make the corresponding inverse transformation to obtain the required analytical solution. The similar calculations can be performed also for the rectangular DOS, $D(\varepsilon)=\frac{\Theta(w / 2-|\varepsilon|)}{w}$, for which the Fourier transform has simple analytical solution $D(t)=\frac{\sin (t w / 2)}{t w / 2}$, where $w$ is the electrode band-width.

In this paper we consider the zero-temperature case and assume a rectangular DOS of the left lead. For the right lead we consider the DOS in the form of two rectangulars with a pseudo-gap between them (relatively low value of DOS, see the inset picture in Fig. 1). In this case analytical formulas for the Fourier transformations of the left and right lead DOS (obtained separatively for each energy region) were used in our calculations and in general 


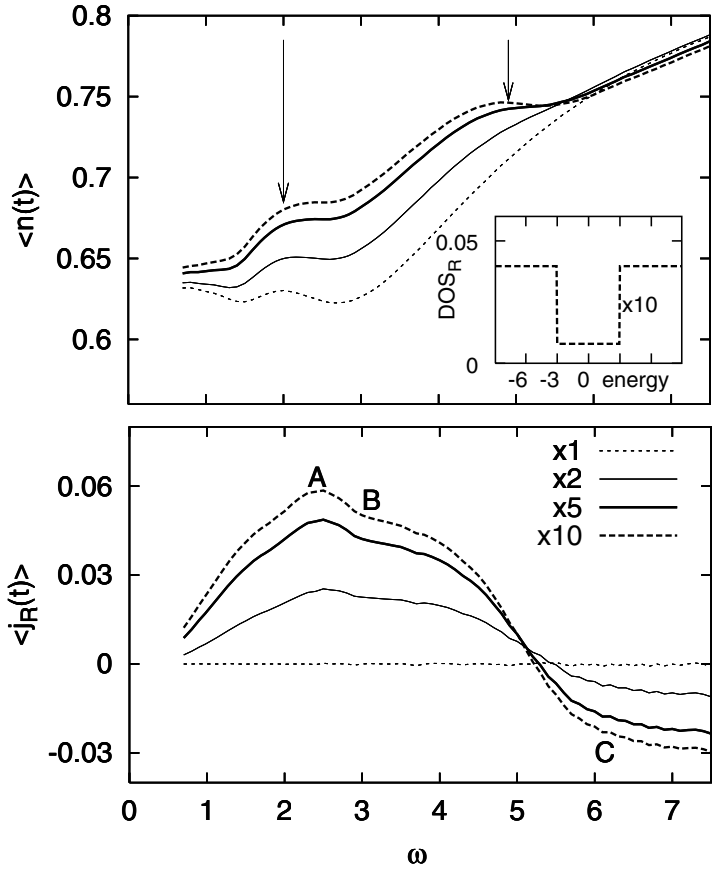

Fig. 1. The averaged QD occupations (upper panel) and the pumped currents flowing from the right lead (bottom panel) as a function of the driving frequency for different left and right DOS structures. The left lead DOS stands for a rectangular, flat shape with nonzero value 0.033 in the energy range -15 to 15 (the band width $w=30$ ). The right lead DOS possesses an energy gap which extends from -3 to 3 with the gap value 2,5 or 10 times smaller than the gapless DOS value (the case $\times 10$ for the right lead DOS is shown in the inset picture in the upper panel). The thin dotted lines, indicated by $\times 1$, correspond to the flat, rectangular-like DOS of the right lead with no energy gap and is the same as the left lead DOS. The arrows and labels A, B, C show specific values of the driving frequency discussed in the text. The other parameter are: $\varepsilon_{0}=-2, \Delta=5, V_{L}=V_{R}=\sqrt{w / 2 \pi}=2.18, E_{F}=0$. The current and energies are expressed in the units of $e \Gamma_{0} / \hbar$ and $\Gamma_{0} \equiv 1$, respectively.

equations (7) and (8) were solved numerically. All energies are expressed in the units of $\Gamma_{0} \equiv 1$, the frequency and current units are $\hbar / \Gamma_{0}$ and $e \Gamma_{0} / \hbar$, respectively. Assuming the QD-lead coupling $\Gamma_{0}=1 \mu \mathrm{eV}$ the corresponding units of the frequency and the current are approximately $1 \mathrm{GHz}$ and $0.2 \mathrm{nA}[1-3]$.

\section{Results: QD occupation and the pumping current}

In the beginning we study the role of an energy gap in the lead DOS on the electron pumping effect through a single level QD coupled with two unbiased leads. The dot is modulated harmonically by a single perturbation in the form of an ac microwave field with the driving frequency $\omega$. In Figure 1 the averaged QD occupation (upper panel) and the pumping current flowing from the right lead (bottom panel) are shown as a function of the driving frequency for different structures of the right lead DOS. Note that the left lead DOS (metallic-like) is modelled as a rectangular structure (with the edges lying far from the QD energy level and the Fermi level) and is fixed in the calculations. The thin dotted lines, indicated by $\times 1$, are obtained for the same both right and left lead DOS i.e. with no energy gaps. As one can see, the QD occupancy possesses a local maximum near $\omega=2$ (indicated by the arrow in the upper panel, thin dotted curve) because in that case one of the QD sideband states with the energy $\varepsilon_{0}+\omega=-2+2=0$ crosses the Fermi energy, $E_{F}=0$. Thus for somewhat larger $\omega$ this sideband state loses its charge (electrons can flow from this state to the empty states of the left and right leads) and in consequence the QD occupancy decreases. For larger driving frequencies the QD charge increases due to the sideband states, $\varepsilon_{0}-n \omega$, which move with $\omega$ deeper and deeper below the Fermi energy and are more occupied. Thus the first local maximum of $\langle n(t)\rangle$ is related with the energy difference between the QD level and the Fermi energy of the system. It is interesting that similar maxima of $\langle n(t)\rangle$ one observes also in the presence of an energy gap in the right lead DOS (three upper curves for $\omega=2$ ). Here we consider the gapped structure in the right lead DOS which extends from -3 to 3 with the value being 2,5 or 10 times smaller than for the gapless DOS value - see the inside figure in the upper panel in Figure 1 for the case $\times 10$. Moreover, the next local maximum of the QD occupation is visible for $\omega \simeq 5$ - see the shorter arrow. However, this maximum appears only for the case of energy-gapped DOS of the right lead, namely, for $\omega=5$ there is the QD sideband state, $\varepsilon_{0}+\omega=-2+5=3$, which crosses the upper edge of the energy gap. For somewhat larger $\omega$ the charge localized at this state, $\varepsilon_{0}+\omega$, can flow with the same probability to the left or to the right electrode and thus the total QD charge locally decreases.

The corresponding pumping currents flowing through the system are depicted in the bottom panel in Figure 1. Note, that in the case of the same structure of the left and right DOS the current does not flow due to the spatial symmetry of the system (thin broken curve, $\times 1$ ). In the presence of the energy pseudo-gap in the right lead DOS electrons are pumped from the right or left lead depending on the driving frequency (solid, thick solid and thick broken curves for $\times 2, \times 5$ and $\times 10$, respectively). As one can see the pumped current depends on the pseudogap DOS value and for deeper gaps the absolute value of the current increases. However, the structure of these curves, except the case of the same $L$ and $R$ DOS $(\times 1)$ are similar. To explain the origin of non-zero current flowing through the unbiased system we analyze in details electron processes which occur for the case $\times 10$ (thick broken curve) for three specific points i.e. for $\omega=2.5$ (maximal current), $\omega=2.9$ (fast decreasing of the current) and $\omega=6$ (negative current) - they are indicated by letters A, B and C, respectively. The energy schemes of the system corresponding to these three cases are shown in Figure 2, panels A, B and C, respectively. On each panel one can see the rectangular left lead DOS with the Fermi energy, $E_{F}$, the right lead DOS with the energy pseudo-gap 


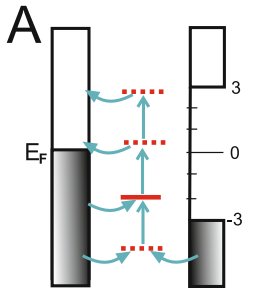

$\omega=2.5$

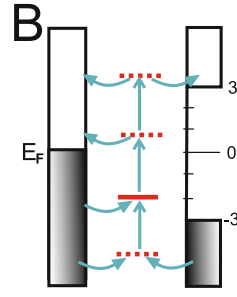

$\omega=2.9$

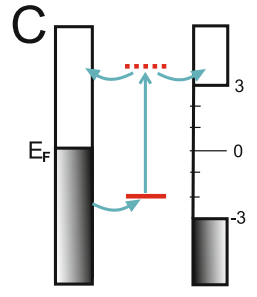

$\omega=6$
Fig. 2. Schematic view of the electron transport processes which occur in the system composed of a gapless left lead, driven QD (with the sideband states) and the right electrode with the pseudo-gap in the DOS structure. The frequencies of the driving fields are $\omega=2.5,2.9$ and 6 , panel A, B and C, respectively. These pictures correspond to points $\mathrm{A}, \mathrm{B}$ and $\mathrm{C}$ shown in the bottom panel in Figure 1.

and the QD energy level $\left(\varepsilon_{0}=-2\right.$, solid lines between leads) with the sideband states $\left(\varepsilon_{0} \pm n \omega\right.$, dashed lines). These states play a crucial role in the pumping current. The bend arrows in Figure 2 indicate the possible electron paths through the system and for sideband states below (above) the Fermi level electrons can tunnel only from the leads to these states (from these states to the leads). As was mentioned above the pumped current is maximal for $\omega=2.5$ (Fig. $2 \mathrm{~A}$ ) and in that case the most probable electron path is $R \rightarrow\left(\varepsilon_{0}-\omega\right) \rightarrow\left(\varepsilon_{0}+\omega\right) \rightarrow L$ i.e. the right lead electron tunnels to the sideband state with the energy $\varepsilon_{0}-\omega$, then absorbs two photons of the external field and forms the PAT state above the Fermi energy, $\varepsilon_{0}+\omega$, and finally it can move with much greater probability to the left electrode (there is very low DOS value in the right lead). Of course there are also other possible paths for electrons in the system which use higher-order, $n>2$, photon processes e.g. $R \rightarrow\left(\varepsilon_{0}-\omega\right) \rightarrow\left(\varepsilon_{0}+2 \omega\right) \rightarrow L$, $L \rightarrow \varepsilon_{0} \rightarrow\left(\varepsilon_{0}+3 \omega\right) \rightarrow L / R$ but they hold with much smaller probabilities. Note that electron processes between the same left or right electrode, such as $L \rightarrow\left(\varepsilon_{0}-\omega\right) \rightarrow$ $\left(\varepsilon_{0}+\omega\right) \rightarrow L, L \rightarrow \varepsilon_{0} \rightarrow\left(\varepsilon_{0}+\omega\right) \rightarrow L$, do not generate the net current. For somewhat larger $\omega$ the current decreases and for $\omega=2.9$ the inflection point is visible, point $\mathrm{B}$ in Figure 1, bottom panel. To explain this effect it is desirable to analyze the energy scheme depicted in Figure 2B. Now the second sideband state with the energy $\varepsilon_{0}+2 \omega$ is beyond the right lead energy gap and the electron paths $L / R \rightarrow\left(\varepsilon_{0}-\omega\right) \rightarrow\left(\varepsilon_{0}+2 \omega\right) \rightarrow L / R$ are equivalent (for electrons there is the same probability to tunnel from these sideband states to the left or to the right electrode). Therefore, for $\omega=2.9$ the state $\varepsilon_{0}+2 \omega$ does not enhance the right current (here the most probable electron path is as before $\left.R \rightarrow\left(\varepsilon_{0}-\omega\right) \rightarrow\left(\varepsilon_{0}+\omega\right) \rightarrow L\right)$. On the other hand, the states with energies $\varepsilon_{0}+2 \omega$ and $\varepsilon_{0}$ can generate small left current i.e. $L \rightarrow \varepsilon_{0} \rightarrow\left(\varepsilon_{0}+2 \omega\right) \rightarrow R$. This process, however, uses the second order QD PAT state, $n=2$, and thus it is less probable than the mentioned above processes where only the first order PAT states are used i.e. for $n= \pm 1$. As one can see in Figure 1 for larger driving frequencies, $\omega>5$, the pumped current changes its direction and flows from the left lead. In that case the most probable electron paths are depicted

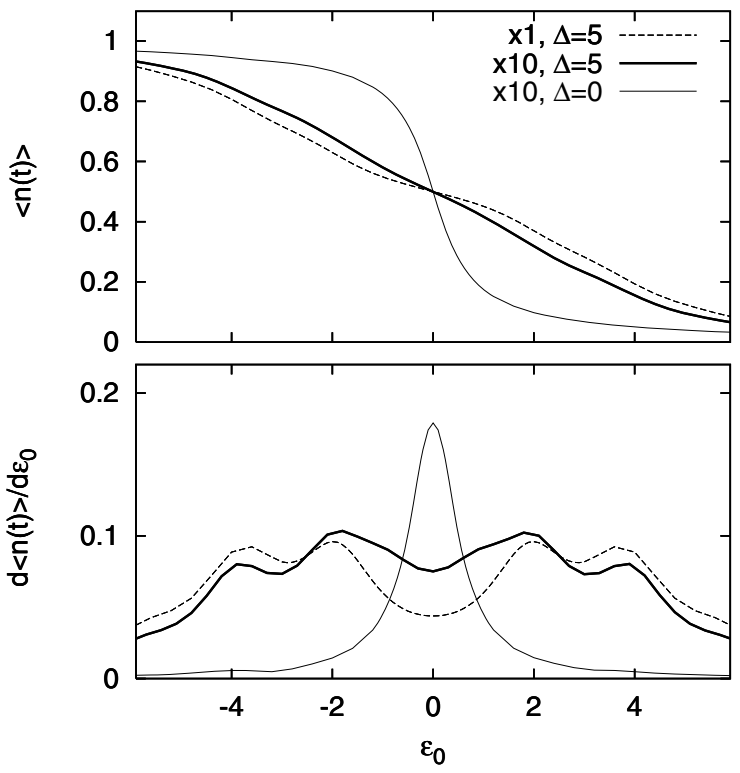

Fig. 3. The averaged QD occupations (upper panel) and their derivatives as a function of $\varepsilon_{0}$ for the rectangular, gapless DOS of the left lead and (i) for the right lead DOS with the energy pseudo-gap in the middle of the band $(\times 10)$ as depicted in the inside figure in Figure 1 and for the static QD, $\Delta=0$ (thin solid curves); (ii) for the same energy-gapped right lead DOS and for the QD driving amplitude $\Delta=5$ (thick solid curves); and (iii) for the rectangular, gapless right lead DOS and $\Delta=5$ (broken curves). The other parameter are the same as in Figure 1, $\omega=2$.

in Figure 2C: $L \rightarrow \varepsilon_{0} \rightarrow\left(\varepsilon_{0}+\omega\right) \rightarrow L / R$ i.e. from the left lead to the QD state $\varepsilon_{0}$, then one-photon adsorption to the PAT state $\varepsilon_{0}+\omega$ takes place and finally to the right lead (transition from the state $\varepsilon_{0}+\omega$ to the left lead is also probable but does not generate the net current).

We can conclude that the lead DOS structure leaves its fingerprints on the average QD charge and pumped current curves (obtained as a function of the driving frequency). For the same left and right leads DOS the current does not flow for the static nor for the driven QD. We have found that electrons are pumped through the system if there are at least one sideband state lying below and the second one lying above the Fermi energy, and additionally, the left and right lead DOS in the vicinity of these states are different (see e.g. the position of the QD states and the left and right DOS in Fig. 2C).

In order to reveal further the role of the right lead energy gap on the QD charge, in Figure 3 we show the occupation of the QD (upper panel) and its derivative (bottom panel) as a function of the QD energy level $\varepsilon_{0}$. For the undriven system, $\Delta=0$, the QD occupation decreases rapidly for $\varepsilon_{0} \simeq E_{F}=0$ and its derivative is characterized by a single peak localized at the Fermi energy, independently on the structure of the right lead DOS (thin solid curves for gapped DOS and very similar for the gapless structure of DOS - not shown here). It means that in the absence of the driving forces the QD charge is determined mainly by the position of $\varepsilon_{0}$ versus 
the Fermi energy. The situation changes in the presence of the periodic harmonic field. The broken curves are obtained for the same gapless left and right electrodes, the case $\times 1$, and for nonzero QD driving amplitude, $\Delta=5$, and the solid curves correspond to the right lead with the energy gap. As one can see the QD occupations decrease with $\varepsilon_{0}$ (broken and thick solid lines) but these curves posses many inflections which are well visible as local peaks on the $d\langle n(t)\rangle / d \varepsilon_{0}$ curves (bottom panel). Interestingly, that these peaks appear for such values of $\varepsilon_{0}$ for which the corresponding QD sideband states cross the Fermi energy i.e. for $\varepsilon_{0} \pm n \omega=E_{F}\left(\varepsilon_{0}= \pm 2, \pm 4, \ldots\right)$. Note that the structure of the right lead DOS is slightly visible on the QD occupation and its derivative curves see small inflection points on $d\langle n(t)\rangle / d \varepsilon_{0}$ for $\varepsilon_{0}= \pm 1$ which correspond to one photon adsorption/emission process near the energies of the gap edges, $\varepsilon_{0}= \pm 3 \mp \omega$. Therefore, in the next studies we answer the question whether the gap structure in DOS can be clearly observed (more evident) on other characteristics of the QD (e.g. on the transconductance).

In Figure 4 we investigate the pumped currents (thick curves) and their derivatives (transconductance, thin curves) as a function of the gate voltage (position of $\varepsilon_{0}$ ). The upper, middle and bottom panels correspond to different energy-gapped structures of the right lead DOS which are shown by the broken curves. For the Fermi energy localized in the middle of the right DOS gap (upper panel, $E_{F}=0$, fully symmetrical energy gap) the current is pumped from the right lead (for $\varepsilon_{0}<E_{F}$ ) and from the left lead (for $\varepsilon_{0}>E_{F}$ ) with the maximal value for $\varepsilon_{0}= \pm 1.8$. The energy scheme of the considered system (for $\varepsilon_{0}=+1.8$, point $\mathrm{A}$ ) is shown in Figure 5A. As one can see electrons tunnel from the left lead to the first-order sideband state with energy $\varepsilon_{0}-\omega$ then adsorb two photons going to the upper first-order sideband state $\varepsilon_{0}+\omega$, and finally can appear in the left or right lead (with the same probability) i.e. $L \rightarrow\left(\varepsilon_{0}-\omega\right) \rightarrow\left(\varepsilon_{0}+\omega\right) \rightarrow L / R$. This process generates the net left current in the system. Note that here a single photon adsorption is the most probable, with the path $L \rightarrow\left(\varepsilon_{0}-\omega\right) \rightarrow \varepsilon_{0} \rightarrow L$, however, $\varepsilon_{0}-\omega$ and $\varepsilon_{0}$ states are effectively coupled only with the left lead (due to the energy pseudo-gap in the right lead) thus this process does not contribute to the net current.

In our above calculations we have investigated the energy-gapped DOS structures of the right lead with the Fermi energy being exactly in the middle of the gap. However, e.g. in dopped semiconductor materials the Fermi energy can be shifted towards the upper or bottom regions of the gap. Therefore, it is interesting to consider such a system paying special attention on the generation of the pumped current. Thus in the middle and bottom panels in Figure 4 we show the results for the right lead DOS with down-shifted energy gap i.e. for the Fermi energy, $E_{F}=0$, lying inside the gap near the upper gap edge, or above the gap, respectively (see the broken curves of the right DOS in Fig. 4). In both considered cases electrons can flow through the system only from the left lead with the maximal value for $\varepsilon_{0}=1.2$ (point $B$ ) and for $\varepsilon_{0}=0.5$

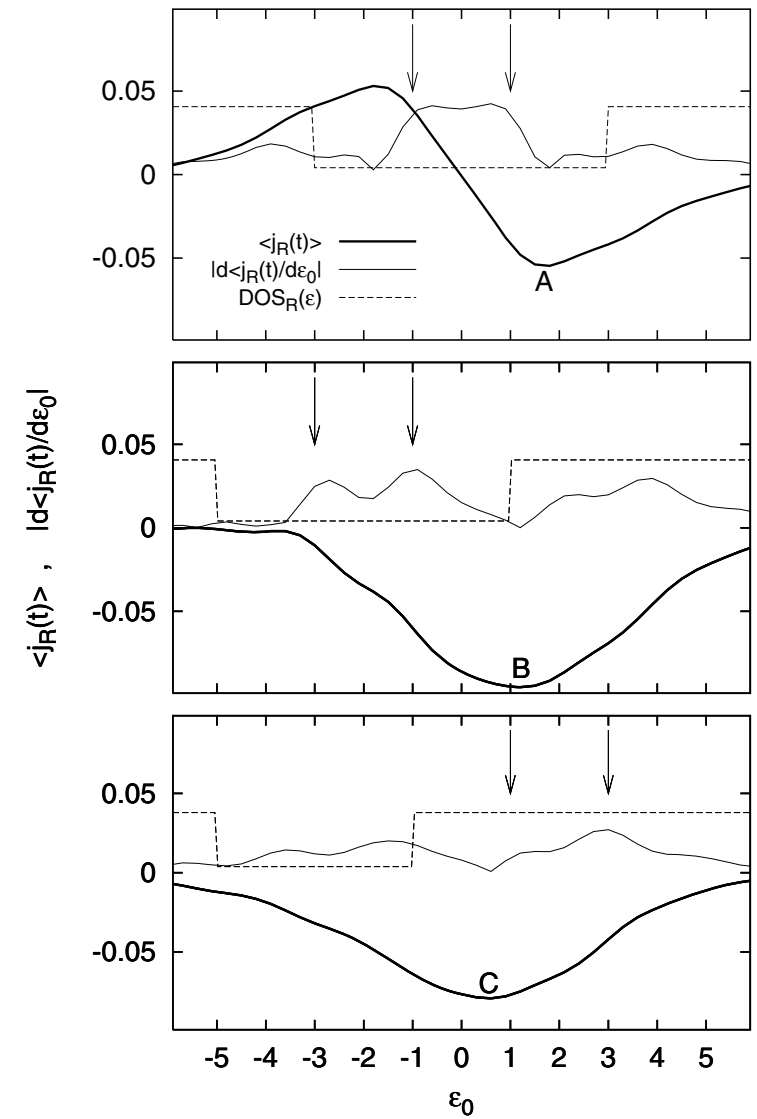

Fig. 4. The pumped current from the right lead (thick solid curves) and the absolute value of the transconductance (thin solid curves) as a function of $\varepsilon_{0}$ for the rectangular, gapless DOS of the left lead and for the right lead DOS (broken curves) with the energy gap in the middle of the band with the gap edges at \pm 3 (upper panel, cf. the inside figure in Fig. 1) and for down-shifted gaps vs. $E_{F}=0$ (middle panel with the gap edges at $-5,+1$ and bottom panel with the gap edges at -5 and -1 ). The arrows and labels A, B, C show specific positions of the QD energy level discussed in the text. The other parameter are the same as in Figure 1, $\omega=2$.

(point C). We explain the current behavior for these cases using the energy schemes indicated in Figures $5 \mathrm{~B}$ and $5 \mathrm{C}$, respectively. For $\varepsilon_{0}=1.2$ (Fig. $5 \mathrm{~B}$ ) the most probable electron path is $L \rightarrow\left(\varepsilon_{0}-\omega\right) \rightarrow \varepsilon_{0} \rightarrow L / R$ i.e. electrons flow from the left lead to the first-order sideband state with energy $\varepsilon_{0}-\omega$, next one-photon absorption takes place and they can tunnel to the right or left electrode. These processes generate the left current which is about two times larger in comparison with the symmetrical gap case with two-photon absorption (upper panel in Fig. 4). As one can see, the similar processes hold also for the gap situated below the Fermi energy (Fig. 5C, for $\varepsilon_{0}=0.5$ ).

The structure of the pumped current curves as a function of $\varepsilon_{0}$ seems rather smooth, however, there are interesting features which are accentuated on the corresponding derivative curves, $d\left\langle j_{R}(t)\right\rangle / d \varepsilon_{0}$ (thin solid lines). The transconductance possesses many peaks - some of them are related to the position of the Fermi energy and they 


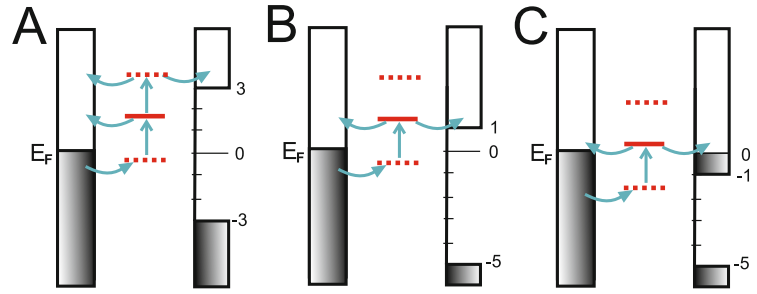

Fig. 5. Schematic view of the electron transport processes which occur in the considered in Figure 4 systems for $\varepsilon_{0}=$ 1.8, 1.2 and 0.5 , panels A, B and C, respectively. These pictures correspond to points $\mathrm{A}, \mathrm{B}$ and $\mathrm{C}$ shown in Figure 4.

appear for $\varepsilon_{0}=E_{F} \pm n \omega$, see e.g. the upper panel in Figure 4 for $\varepsilon_{0}= \pm 2, \pm 4, \ldots$, cf. [43]. However, there are also other peaks which are indicated by the arrows in all panels. It seems that they are related to the structure of the right lead DOS because they appear for $\varepsilon_{0}= \pm 1$, i.e. for $\varepsilon_{0}$ which differs by the energy $\pm \omega$ from the gap edges, $\varepsilon_{0}=3-\omega$ and $\varepsilon_{0}=-3+\omega$ (upper panel), for $\varepsilon_{0}=-1,-3$, i.e. for $\varepsilon_{0}=1-\omega$ and $\varepsilon_{0}=-5+\omega$ (middle panel) and for $\varepsilon_{0}=1,3$ i.e. for $\varepsilon_{0}=-1+\omega$ and $\varepsilon_{0}=-1+2 \omega$ (bottom panel). Thus it is possible to find the energy-gap parameters from the transconductance peaks. In order to confirm the origin of these peaks further investigation of the pumping current is needed.

In Figure 6 we consider mono-parametric pumping through a QD for two different DOS structures of the right lead i.e. with very sharp and fuzzy (linear-dependent) gap edges (see the broken curves). The upper (bottom) panel corresponds to the limit of strong (weak) coupling parameters, $V_{L}=V_{R}=2.18\left(V_{L}=V_{R}=1\right)$. Note that the upper curve in the upper panel, obtained for the rectangulargap structure, is exactly the same as that one shown in Figure 4, upper panel. We are interested in the transconductance peaks (which are indicated by the arrows) and their modifications due to the structure of the energy gap edges. As one can see for the rectangular gap in DOS these peaks are situated for the energies which differ by $\pm \omega$ from the gap edges and appear for both strong and weak couplings (upper and bottom panel, upper curves). However, if there are no sharp edges of the gap these peaks disappear which is better visible in the bottom panel (weak couplings). Note that there are also other peaks which are related to the Fermi energy - they appear for $\varepsilon_{0}=E_{F} \pm n \omega= \pm 2, \pm 4, \ldots$ independently of the energy gap edges. It confirms that the considered transconductance peaks are strictly related to the gap structure in lead DOS. Thus, the pumped current and the transconductance of the metal-QD-(semiconductor-like) system provide useful information about the DOS structure and the system Fermi energy. Mono-parametric pumping can be also useful in determination of other structures of the lead DOS with peaks or surface states.

\section{Conclusions}

We have considered mono-parametric pumping in the unbiased system composed of the left lead (with the flat

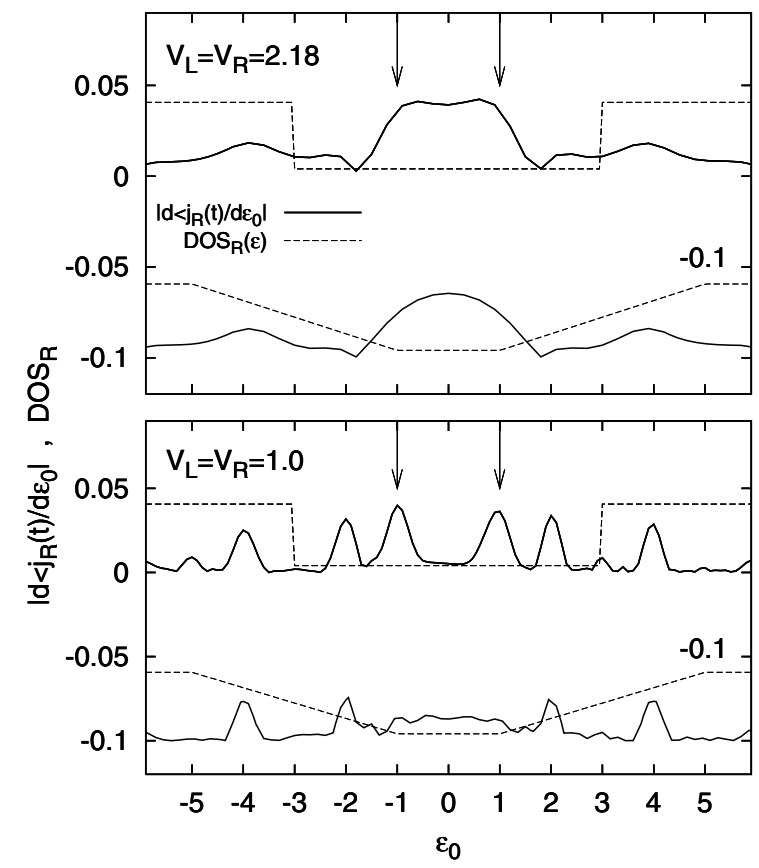

Fig. 6. The transconductance (thin solid curves) as a function of $\varepsilon_{0}$ for the coupling parameters $V_{L}=V_{R}=2.18$ (upper panel) and $V_{L}=V_{R}=1$ (bottom panel) and for different right lead DOS structures (broken lines): (i) with the energy gap which extends from -3 to 3 with sharp edges (upper curves) and for the linear-dependence of the gap energy in the ranges $(-5,-1)$ and $(1,5)$ - bottom curves, they are shifted down by 0.1 for better visualization. The arrows show the transconductance peaks discussed in the text. The other parameter are the same as in Figure 4.

DOS), a single level harmonically driven quantum dot and the right lead described by DOS with the energy pseudogap. The model tight-binding Hamiltonian and the evolution operator technique were used in our calculations. The QD occupancy and the pumped current were expressed by the appropriate matrix elements of the evolution operator for which the integro-differential equations were solved numerically.

It was shown that if the spatial symmetry of the system is broken (one lead is characterized by the energy-gapped structure of DOS) the single-parameter charge pump can exist. We have also found that the direction of the pumping current flowing through the QD depends on the external field driving frequency. We have analyzed in details the role of the position of the sideband energies on the net current. As a main result of this work we have found that the pumped current appears in the system if there are at least two QD sideband states - one of them lying above and the second one lying below the Fermi energy and the left and right lead DOS in the vicinity of these states are different. Moreover, we have shown that the structure of the lead's DOS is visible on the average QD charge as well as on the pumped current characteristics. The gapped DOS structure is also reflected on the transconductance curves. Thus mono-parametric pumping through a QD allows us 
to determine the lead DOS parameters directly form the measurement of the pumped current.

This work has been partially supported by the National Science Center, Poland, Grant No. 2014/13/B/ST5/04442.

\section{References}

1. T.H. Oosterkamp, K.P. Kouwenhoven, A.E. Koolen, N.C. van der Vaart, C. Harmans, Phys. Rev. Lett. 78, 1536 (1997)

2. L.P. Kouwenhoven, A.P. Johnson, N.C. van der Vaart, A. van der Enden, C. Hermans, C.T. Foxon, Z. Phys. B 85, $381(1991)$

3. P. McEuen, L.P. Kouwenhoven, S. Jauhar, J. Orenstein, K. McCormick, D. Dixon, Yu.V. Nazarov, N.C. van der Vaart, C. Foxon, Nanotechnology 7, 406 (1996)

4. R.H. Blick, R.J. Haug, J. Weis, D. Pfannkuche, K. von Klitzing, K. Eberl, Phys. Rev. B 53, 7899 (1996)

5. S.K. Watson, R.M. Potok, C.M. Marcus, V. Umansky, Phys. Rev. Lett. 91, 258301 (2003)

6. T. Kwapiński, R. Taranko, Physica E 18, 402 (2003)

7. G. Platero, R. Aguado, Phys. Rep. 395, 1 (2004)

8. P.W. Brouwer, Phys. Rev. B 58, R10135 (1998)

9. M. Seo, Ye-Hwan Ahn, Y. Oh, Y. Chung, S. Ryu, H.S. Sim, I.-H. Lee, M.-H. Bae, N. Kim, Phys. Rev. B 90, 085307 (2014)

10. M. Switkes, C.M. Marcus, K. Campman, A.C. Gossard, Science 283, 1905 (1999)

11. B. Roche, R.-P. Riwar, B. Voisin, E. Dupont-Ferrier, R. Wacquez, M. Vinet, M. Sanquer, J. Splettstoesser, X. Jehl, Nat. Commun. 4, 1581 (2012)

12. V. Gasparian, B. Altshuler, M. Ortuno, Phys. Rev. B 72, 195309 (2005)

13. M. Mahmoodian, L. Braginsky, M. Entin, Phys. Rev. B 74, 125317 (2006)

14. E. Faizabadi, F. Ebrahimi, J. Phys.: Condens. Matter 16, $1789(2004)$

15. S. Das, S. Rao, Phys. Rev. B 71, 165333 (2005)

16. B. Wang, J. Wang, H. Guo, Phys. Rev. B 65, 073306 (2002)

17. E. Prada, P. San-Jose, H. Schomerus, Phys. Rev. B 80, 245414 (2009)

18. B. Abdollahipour, R. Mohammadkhani, J. Phys.: Condens. Matter 26, 085304 (2014)

19. L.H. Ingaramo, L. Foa Torres, Appl. Phys. Lett. 103, $123508(2013)$

20. S.K. Watson, R.M. Potok, C.M. Marcus, V. Umansky, Phys. Rev. Lett. 91, 258301 (2003)

21. T. Kwapiński, R. Taranko, J. Phys.: Condens. Matter 23, 405301 (2011)

22. Y. Avishai, D. Cohen, N. Nagaosa, Phys. Rev. Lett. 104, 196601 (2010)

23. P.J. Leek, M.R. Buitelaar, V.I. Talyanskii, C.G. Smith, D. Anderson, G.A.C. Jones, J. Wei, D.H. Cobden, Phys. Rev. Lett. 95, 256802 (2005)
24. S. Kohler, J. Lehmann, P. Hänggi, Phys. Rep. 406, 379 (2005)

25. J. Lehmann, S. Kohler, P. Hänggi, A. Nitzan, Chem. Phys. 118, 3283 (2003)

26. T. Kwapiński, S. Kohler, P. Hänggi, Ukr. J. Phys. 55, 85 (2010)

27. M. Rey, M. Strass, S. Kohler, P. Hänggi, F. Sols, Phys. Rev. B 76, 085337 (2007)

28. L. Foa Torres, Phys. Rev. B 72, 245339 (2005)

29. M.G. Vavilov, L. DiCarlo, C.M. Marcus, Phys. Rev. B 71, 241309(r) (2005)

30. L. DiCarlo, C.M. Marcus, J.S. Harris, Phys. Rev. Lett. 91, $246804(2003)$

31. B. Kaestner, V. Kashcheyevs, S. Amakawa, M.D. Blumenthal, L. Li, T.J.B.M. Janssen, G. Hein, K. Pierz, T. Weimann, U. Siegner, H.W. Schumacher, Phys. Rev. B 77, 153301 (2008)

32. T. Aref, V.F. Maisi, M.V. Gustafsson, P. Delsing, J.P. Pekola, Europhys. Lett. 96, 37008 (2011)

33. L. Foa Torres, H. Calvo, C. Rocha, G. Cuniberti, Appl. Phys. Lett. 99, 092102 (2011)

34. P. San-Jose, E. Prada, S. Kohler, H. Schomerus, Phys. Rev. B 84, 155408 (2011)

35. C.A. Perroni, A. Nocera, V. Cataudella, Europhys. Lett. 103, 58001 (2013)

36. Y. Zhou, M.W. Wu, Phys. Rev. B 86, 085406 (2012)

37. P. San-Jose, E. Prada, H. Schomerus, S. Kohler, Appl. Phys. Lett. 101, 153506 (2012)

38. M. Strass, P. Hänggi, S. Kohler, Phys. Rev. Lett. 95, 130601 (2005)

39. T.B. Grimley, V. Bhasu, K.L. Sebastian, Surf. Sci. 121, 305 (1983)

40. R. Taranko, T. Kwapiński, E. Taranko, Phys. Rev. B 69, $165306(2004)$

41. T. Kwapiński, J. Phys.: Condens. Matter 22, 295303 (2010)

42. Y.-Q. Zhou, R.-Q. Wang, L. Sheng, B. Wang, D.Y. Xing, Phys. Rev. B 78, 155327 (2008)

43. A.-P. Jauho, N.S. Wingreen, Y. Meir, Phys. Rev. B 50, 5528 (1994)

44. T. Kwapiński, J. Phys.: Condens. Matter 19, 176218 (2007)

45. J. Maciejko, J. Wang, H. Guo, Phys. Rev. B 74, 085324 (2006)

46. T. Kwapiński, R. Taranko, E. Taranko, Phys. Rev. B 66, 035315 (2002)

47. D.M. Newns, Phys. Rev. B 178, 1123 (1968)

48. S. Tsuneyuki, N. Shima, M. Tsukada, Surf. Sci. 186, 26 (1987)

Open Access This is an open access article distributed under the terms of the Creative Commons Attribution License (http://creativecommons.org/licenses/by/4.0), which permits unrestricted use, distribution, and reproduction in any medium, provided the original work is properly cited. 